\title{
Estudio del Comportamiento Fluido-Dinámico de un Agitador a Escala Reducida Mediante Simulación Numérica
}

\author{
José C. Chambergo*, Quino Valverde, Alex A. Pachas y Herbert Yépez \\ Grupo de Investigación Asistida por Computadora INACOM, Pontificia Universidad Católica del Perú, \\ PUCP, Dpto. de Ingeniería, Secc. Ingeniería Mecánica, Av. Universitaria 1801, Lima 32-Perú \\ (e-mail: jcchambergo@pucp.edu.pe) \\ * Autor a quien debe ser dirigida la correspondencia
}

Recibido Sep. 25, 2016; Aceptado Dic. 9, 2016; Versión final Mar. 10, 2017, Publicado Jun. 2017

\begin{abstract}
Resumen
Se presenta el estudio del comportamiento de un agitador con impulsor hidroala (hydrofoil), usado para mezclar agua con partículas polimetálicas en la industria minera, mediante simulación numérica con software de dinámica de fluidos computacional (CFD). La mezcla tiene densidad promedio de $1.623 \mathrm{~kg} / \mathrm{m}^{3}$, viscosidad aparente de $0.065 \mathrm{~kg} / \mathrm{m} \cdot \mathrm{s}$ y fue modelada como un líquido homogéneo no-newtoniano. En la simulación el problema se resuelve mediante un modelo homogéneo Euler-Euler para flujo bifásico de superficie libre en estado estacionario, utilizando el modelo de turbulencia $k-\varepsilon$. Se obtuvo la curva de potencia, que relaciona el número de potencia $(\mathrm{Np})$ con el número de Reynolds $(\mathrm{Re})$, a partir de la curva de torque del impulsor en función de su velocidad angular entre 200 y $700 \mathrm{rpm}$. Se encontró que Np converge asintóticamente a 0.32 en régimen turbulento, valor que está en correspondencia con los resultados experimentales obtenidos en el presente estudio y los disponibles en la literatura.
\end{abstract}

Palabras clave: agitador; número de potencia; impulsor hidroala; CFD; mezcladores

\section{Study of the Fluid-Dynamic Behavior of a Reduced Scale Stirred Tank through Numerical Simulation}

\begin{abstract}
This paper presents the study of the behavior of a stirred tank with hydrofoil impeller, used to mix water with polymetallic particles in the mining industry, through numerical simulation with CFD software. The mixture has an average density of $1.623 \mathrm{~kg} \mathrm{/} \mathrm{\textrm {m } ^ { 3 }}$ and an apparent viscosity of $0.065 \mathrm{~kg} / \mathrm{m}-\mathrm{s}$, and was modeled as a non-newtonian homogeneous liquid. The homogeneous Euler-Euler model for free surface biphasic flow in steady state is solved in the simulation, using the $k-\varepsilon$ turbulence model. The power curve, which correlates the power number $(\mathrm{Np})$ with the Reynolds number $(\mathrm{Re})$, was obtained from the torque curve of the impeller versus its angular velocity between 200 and $700 \mathrm{rpm}$. It was found that $\mathrm{Np}$ asymptotically converges to 0.32 in turbulent regime, a value that is in agreement with experimental results obtained in the present study and those available in the literature.
\end{abstract}

Keywords: impeller; power number; hydrofoil impeller; CFD; stirred tank 


\section{INTRODUCCIÓN}

Los tanques agitadores son comunes e importantes para diferentes aplicaciones industriales tales como: petroquímica, farmacéutica, biotecnología, minera entre otros (Qingbai y Gance, 2010; Joshi et al., 2011; Yapici, et al., 2008) El proceso de agitación en el presente caso se refiere a forzar el fluido por medios mecánicos (eje e impulsor) a moverse de forma circulatoria alrededor del eje del tanque. El movimiento circulatorio del fluido impacta sobre deflectores que están dentro del tanque generando una disminución del tamaño del remolino y mejorando la eficiencia en la agitación del fluido. (Harnby et al., 2000; Yang y Zhou, 2015). Según (Patankar S., 2002), hay una multitud de escalas temporales y geométricas asociadas al flujo en el interior de máquinas que operan con fluidos. Estas son descritas en tres categorías típicas: las de orden de magnitud del diámetro (o tamaño) de la máquina, fluctuaciones periódicas asociadas al paso de álabe y perturbaciones de pequeña escala, asociadas con las estructuras turbulentas del flujo.

Para el diseño de tanques agitadores, se han establecido técnicas experimentales con el propósito de conocer parámetros de funcionamiento, el consumo de potencia, homogeneidad de fluidos, entre otros (Zwietering, 1958; Wu et al., 2006; Wu et al., 2010a; Wu et al., 2010b; Ibrahim, 2012). Además, a partir de ensayos experimentales se logra obtener correlaciones empíricas mediante la relación entre variables y parámetros de funcionamiento. Sin embargo, la complejidad y diversidad de los fenómenos de fluctuaciones de presión y gradientes de velocidad en un análisis experimental conlleva algunas incertidumbres en los resultados. Profundizar en el conocimiento del flujo de fluidos en base exclusivamente a esta metodología puede resultar costoso e incluso impracticable. Desde la década de los 70 , el uso de herramientas computacionales para el desarrollo del análisis del flujo de fluidos y sus fenómenos se ha ido incrementando gracias al mejoramiento de la tecnología computacional y el desarrollo de técnicas numéricas (Shah et al., 2013; Fernández, 2012). La Dinámica de Fluidos Computacional (CFD del inglés Computational Fluid Dynamics) es una herramienta muy útil para analizar en detalle el fenómeno de la turbulencia en tanques agitadores, los cuales son comúnmente usados en procesos industriales (Jaworski y Zakrzewska, 2002; Montante et al., 2001; Gabriele et al., 2009).

Las técnicas de CFD, permiten predecir el desarrollo del flujo de fluido, tanto en modelos de laboratorio como a escala industrial directamente, visualizando parámetros de interés sea en estado estacionario o transitorio (Kehn, 2013). Además, mediante estas técnicas se pueden estudiar nuevos diseños en diversos escenarios de operación a un costo relativamente bajo; no obstante, siempre es necesario validar la simulación numérica mediante datos experimentales (Chen y Bai, 2017). Entre las diversas aplicaciones industriales, los agitadores con impulsores axiales son los de mejor desempeño en la suspensión de sólidos y mezcla, procesos frecuentes y muy importantes en la industria minera. En particular, los impulsores tipo hydrofoil son de especial interés por ser los de menor consumo de energía en la aplicación de la suspensión de sólidos (Spogys y Nunhez, 2016; Harnby et al., 2000). Sin embargo, la mayoría de los estudios de agitadores realizados mediante CFD se han enfocado en el modelamiento de flujos generados por dos tipos de impulsores, Rushton y PBT (Pitched blade turbines), muchos de ellos con agua como fluido de operación (Joshi et al., 2011).

En el presente trabajo se estudia mediante simulación numérica CFD el comportamiento de un agitador modelo a escala reducida con impulsor tipo hydrofoil que opera con una mezcla de agua y pulpa polimetálica, como parte de un proyecto de investigación en colaboración con una empresa metalmecánica que fabrica equipos para la industria minera. El objetivo es determinar el número de Potencia $(N p)$ del impulsor en régimen turbulento. Se determina el torque en estado estacionario en el impulsor con respecto a la velocidad angular de operación; a partir de estos resultados se obtiene la curva de Potencia, que relaciona el número de Potencia con el número de Reynolds $(R e)$. Los resultados de la simulación se contrastan con resultados experimentales obtenidos en el presente estudio y con resultados disponibles en la literatura.

\section{MODELOS MATEMÁTICOS UTILIZADOS EN LA SIMULACIÓN}

El problema del flujo de fluidos se describe mediante ecuaciones matemáticas, tales como las ecuaciones de balance de cantidad de movimiento de Navier Stokes. Estas son ecuaciones diferenciales cuya solución se puede aproximar empleando métodos numéricos como el método de los Volúmenes finitos, por ejemplo. Tal como se ha señalado en la Introducción, el fenómeno involucra escalas cuyos órdenes de magnitud geométrica son significativamente diferentes, lo que en aplicaciones industriales generales implicaría el uso de mallas excesivamente finas en comparación con las dimensiones macroscópicas del problema. En consecuencia, para considerar el efecto de la turbulencia se opta por el uso de modelos de aproximación que permitan resultados aceptables según la aplicación concreta que se aborda. Por razones similares, se modelan matemáticamente otros fenómenos y comportamientos complejos. En este trabajo se emplea ANSYS - CFX v 17.2, software comercial CFD para la simulación del problema aplicando una serie modelos de aproximación (ANSYS, 2016). 


\section{Modelo de turbulencia $k-\varepsilon$}

El modelo $k-\varepsilon$ es uno de los más ampliamente utilizados para el modelamiento de los efectos turbulentos en diversas aplicaciones industriales por su estabilidad computacional, incluso en fenómenos complejos (Aubin et al., 2004; Paul et al., 2004). A partir de la descomposición de la velocidad $\boldsymbol{U}=\overline{\boldsymbol{U}}+\boldsymbol{u}^{\prime}$, en componentes promedio $\overline{\boldsymbol{U}}$ y fluctuante $\boldsymbol{u}^{\prime}$ respectivamente, se resuelve la denominada ecuación de Reynolds Navier Stokes promediada (RANS, por sus siglas en inglés), cuya expresión para la componente $i$ de la velocidad es:

$$
\frac{\partial}{\partial x_{j}}\left(\rho \bar{U}_{i} \bar{U}_{j}\right)=-\frac{\partial p}{\partial x_{i}}+\frac{\partial}{\partial x_{j}}\left[\mu\left(\frac{\partial \bar{U}_{i}}{\partial x_{j}}+\frac{\partial \bar{U}_{j}}{\partial x_{i}}-\frac{2}{3} \frac{\partial \bar{U}_{k}}{\partial x_{k}} \delta_{i j}\right)\right]+\frac{\partial}{\partial x_{j}}\left(-\rho \overline{u_{\imath}^{\prime} u_{\jmath}^{\prime}}\right)+g_{i}
$$

donde: $x_{i}$ representa la coordenada espacial $i, \bar{U}_{l}$ y $u_{i}^{\prime}$ son respectivamente las componentes promediada y fluctuante de velocidad en dirección $i, p$ es la presión, $\mu$ es la viscosidad, $\delta_{i j}$ representa la función delta de Kronecker, $g_{i}$ es la componente de la gravedad en dirección $i$. La barra superior indica términos promediados.

Esta ecuación es similar a la ecuación de Navier Stokes, salvo que en la ecuación (1) aparece un término adicional $-\rho \overline{u_{i}^{\prime} u_{j}^{\prime}}$, denominado tensor de esfuerzos de Reynolds, y que los demás términos dependen de componentes promediadas en el tiempo. Además, para resolver el tensor de esfuerzos de Reynolds, el modelo introduce la definición de dos variables adicionales: la energía cinética de disipación $k$ y el ratio de disipación turbulenta $\varepsilon$, en base a la observación de flujos turbulentos y constantes empíricas (Paul et al., 2004). Este modelo permite captar adecuadamente los efectos de la turbulencia en régimen turbulento, a condición de una buena calidad de malla en la capa límite; cerca de las paredes, la malla debe tener un refinamiento de 10 a 15 capas en dirección normal (Kim, 1995). Al evaluar los resultados, se debe verificar que en la primera de estas capas la altura adimensional $y^{+}$debe ser cercana a la unidad (Fernández, 2012; Versteeg y Malalasekera, 2007).

\section{Enfoque Euler-Euler del Flujo multifásico}

La mezcla de agua con partículas de pulpa polimetálica en el agitador es un fluido de comportamiento no newtoniano, con densidad promedio y viscosidad aparente conocidas. Como simplificación, esta mezcla se modela en la simulación como un líquido homogéneo. De acuerdo con lo anterior, en el modelo del problema están presentes dos fases: mezcla (líquida) y aire (gas) circundante.

En el enfoque de modelado Euler-Euler las fases se tratan como continuas e interpenetrantes y se asume que existen en todo el dominio. Dado que el volumen de una fase no puede ser ocupado por el de otra, se introduce el concepto de fracción de volumen. La fracción de volumen es función de la posición y dependiente del tiempo y la suma de las fracciones de volumen de las fases es igual a uno. En el enfoque Euler-Euler general, las fases se tratan por separado y las ecuaciones se resuelven para cada fase. Además, se debe resolver una ecuación para la fracción de volumen de cada fase. Sin embargo, en la presente aplicación se emplea el modelo Homogéneo de superficie libre incluido en ANSYS-CFX 17.2, que asume como aproximación que no hay deslizamiento relativo entre fases y por tanto las velocidades de las fases son iguales en todo el dominio $\boldsymbol{U}_{l}=\boldsymbol{U}_{g}=\boldsymbol{U}$, donde: $\boldsymbol{U}_{l}$ y $\boldsymbol{U}_{g}$ son los campos vectoriales de velocidad correspondientes a las fases líquida y gas, respectivamente. De esta manera, es necesario resolver solo un campo de velocidad $\boldsymbol{U}$ y una ecuación de transporte para la fracción de volumen de una de las fases (Gidaspow, 1994), por ejemplo para la fracción de volumen de la fase líquida $r_{l}$ :

$$
\frac{\partial}{\partial t}\left(r_{l}\right)+\nabla \cdot\left(r_{l} \boldsymbol{U}\right)=0
$$

Asimismo, la densidad $\rho$ y la viscosidad $\mu$ en la ecuación (1) quedan definidas en función de las correspondientes propiedades de las fases (Gidaspow, 1994), según las ecuaciones:

$$
\begin{aligned}
& \rho=\sum_{\alpha=1}^{N_{P}} r_{\alpha} \rho_{\alpha}=r_{l} \rho_{l}+r_{a} \rho_{a} \\
& \mu=\sum_{\alpha=1}^{N_{P}} r_{\alpha} \mu_{\alpha}=r_{l} \mu_{l}+r_{a} \mu_{a}
\end{aligned}
$$




\section{Modelo de fluido no newtoniano de Bingham}

El comportamiento no newtoniano de la mezcla (fase líquida) se puede representar mediante modelos en los cuales la viscosidad aparente es variable en función de los esfuerzos cortantes en el fluido, a diferencia del comportamiento newtoniano, en el que la relación entre los esfuerzos cortantes y la velocidad de cizallamiento es proporcional a la viscosidad, como propiedad del fluido de valor constante (Constantinescu, 1995). En el presente trabajo se emplea el modelo de Bingham, que se define mediante la ecuación (5) para la viscosidad dinámica aparente:

$$
\mu_{l}=\left(\tau_{Y} / \dot{\gamma}\right)+K
$$

donde: $\mu_{l}$ : viscosidad dinámica o viscosidad aparente de la mezcla, $\tau_{Y}$ : esfuerzo cortante de fluencia, $\dot{\gamma}$ : velocidad de cizallamiento, $K$ : índice de consistencia de la viscosidad. Los valores correspondientes a la mezcla considerada mediante este modelo son: $\tau_{Y}=1.75 \mathrm{~Pa}, K=0.065 \mathrm{~kg} / \mathrm{m} \cdot \mathrm{s}$. La densidad se considera constante y de valor igual al promedio de la mezcla, $1,623 \mathrm{~kg} / \mathrm{m}^{3}$.

\section{PROCEDIMIENTO PARA LA SIMULACIÓN NUMÉRICA}

Se estudia un agitador modelo, fabricado a escala reducida 1:4 con respecto a un agitador industrial fabricado por una empresa metalmecánica. Se realizan simulaciones en estado estacionario en el rango de velocidades de operación de 200 a 700 rpm, obteniéndose el torque en el impulsor, las líneas de corriente y los vectores de velocidad. Con la finalidad de contrastar los resultados de la simulación con resultados experimentales, se realizan en el agitador modelo ensayos de laboratorio en los cuales se determina el torque en el eje del impulsor a partir de lo cual se genera la curva de Potencia.

\section{Modelo geométrico}

En la Fig. 1 se presentan la sección frontal y la vista superior del agitador modelo en el que se realizan las simulaciones y los ensayos de laboratorio.
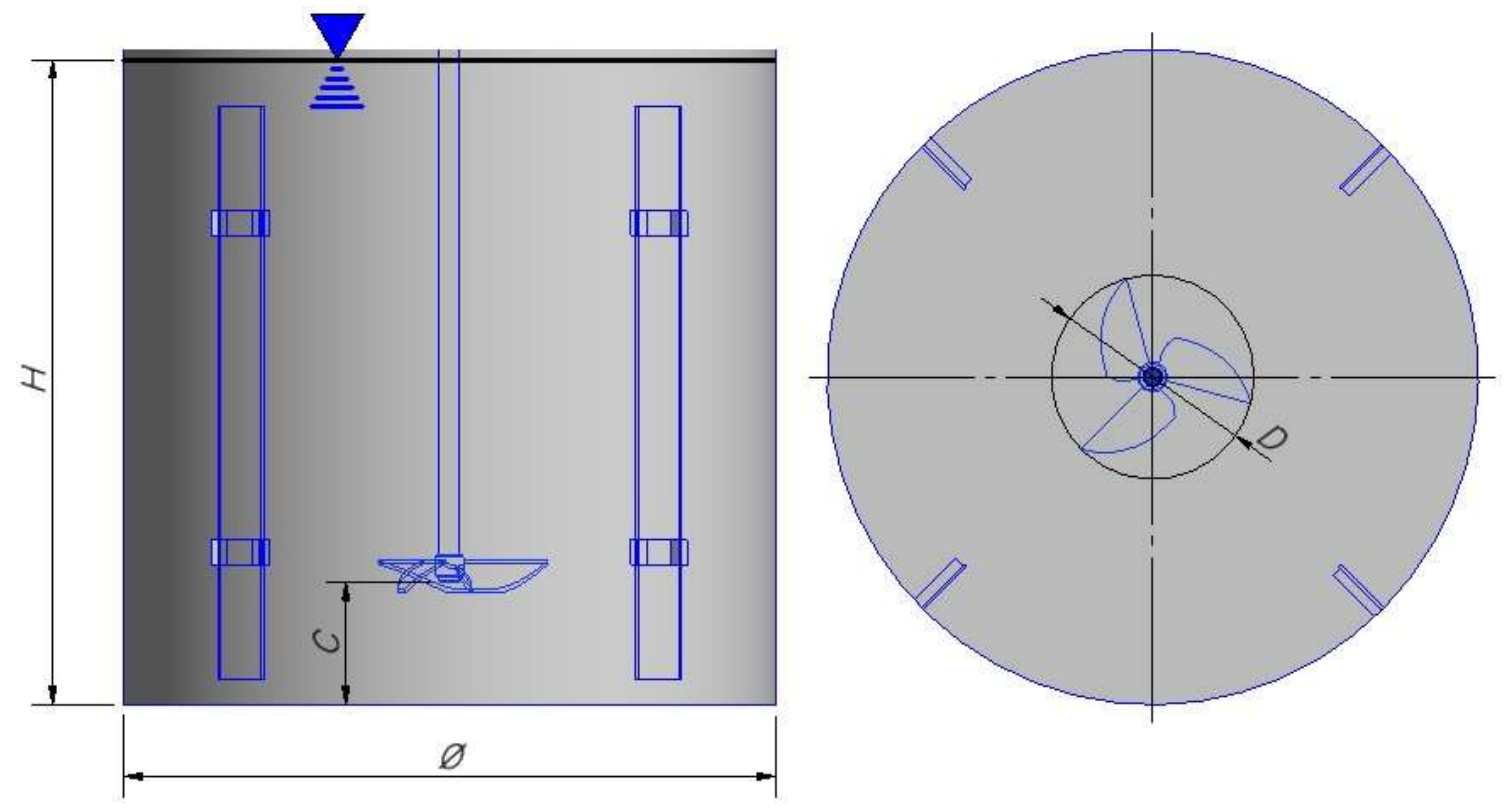

Fig. 1: Agitador a escala reducida y dimensiones principales

En la Fig. 2 se muestra el impulsor de tipo hydrofoil de 3 álabes, con la indicación del ángulo de ataque $\alpha^{\prime}$ de 15․ El agitador consta básicamente de un tanque de forma cilíndrica que alberga la mezcla, 4 deflectores dispuestos en las paredes internas del recipiente y un impulsor axial que gira conectado a un eje vertical. La figura muestra las dimensiones principales del agitador $\phi=610 \mathrm{~mm}$ (diámetro interno del recipiente), $H=600 \mathrm{~mm}$ (altura de la superficie libre de la mezcla en condición de reposo), $D=190 \mathrm{~mm}$ (diámetro del impulsor), y $C=114 \mathrm{~mm}$ (holgura entre la superficie inferior y el fondo del tanque). Se han considerado redondeos con la finalidad de suavizar las aristas en las superficies de los álabes y los deflectores. 


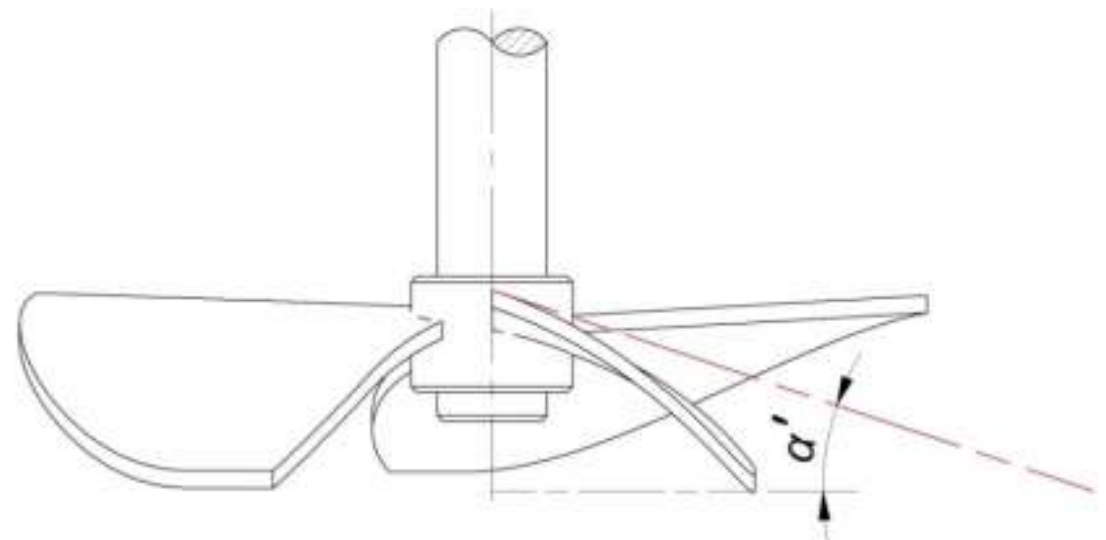

Fig. 2: Impulsor con indicación del ángulo de ataque de los álabes

El modelo considera el dominio ocupado por los fluidos subdividido en dos subdominios, uno rotativo y otro estacionario. Asociado a esta subdivisión se emplea el modelo MRF (por las siglas en inglés de Multiple reference frame), que considera un marco de referencia rotativo que gira junto con el impulsor (Luo, 1994). El subdominio rotativo, designado como I en la Fig. 3, corresponde al espacio encerrado dentro de una superficie cilíndrica alrededor del impulsor. El subdominio estacionario, designado como $T$, corresponde al resto del espacio en el tanque. En esta figura se aprecian también los elementos $A$ y $D$, que son respectivamente: el conjunto eje-impulsor y los 4 deflectores; estos son elementos sólidos y por tanto no forman parte del dominio o espacio ocupado por fluido.

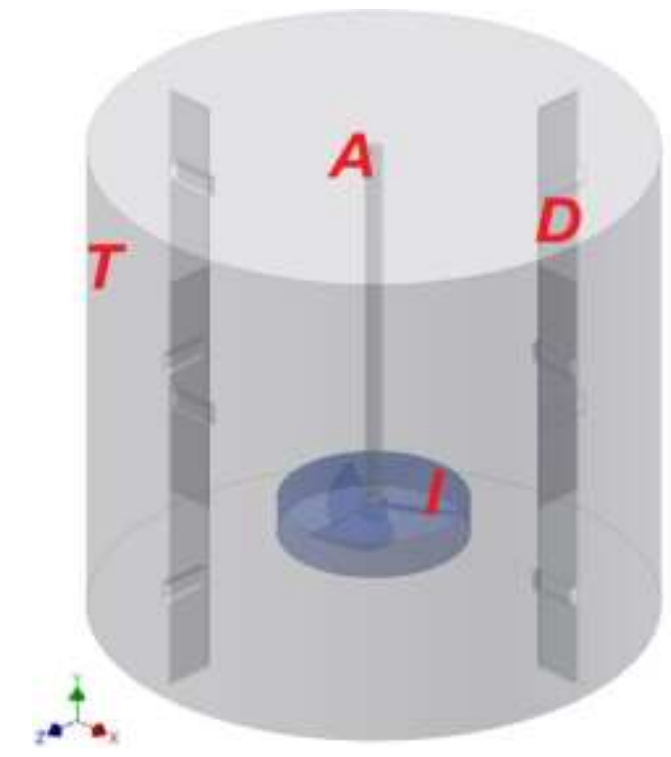

Fig. 3: Subdivisión del dominio: $I$ : subdominio rotativo y $T$ : subdominio estacionario. $A$ (conjunto eje-impulsor) y $D$ (4 deflectores) son elementos sólidos que no son parte de estos subdominios.

\section{Discretización del modelo geométrico}

La malla se genera mediante el programa Ansys Meshing en el dominio ocupado por los fluidos (Fig. 4a). Para acoplar los subdominios rotativo y estacionario se usa el modelo General Connection del programa ANSYS CFX (ANSYS, 2016), que conecta los subdominios de manera apropiada en sus interfaces. En general, los elementos impuestos para la malla son tetraedros, con un tamaño de elementos promedio de $20 \mathrm{~mm}$ en el subdominio estacionario y de $7.5 \mathrm{~mm}$ en el subdominio rotativo. Se obtienen 1'395,185 elementos y 403,349 nodos en el subdominio estacionario y 107,460 elementos y 31,337 nodos en el subdominio rotativo, haciendo un total de 1'502,645 elementos y 434,686 nodos; se alcanza una calidad de malla de $78.58 \%$ y un valor de calidad ortogonal de los elementos de 0.851 . Cerca de las superficies de los sólidos (árbol, impulsor, deflectores y paredes del tanque) se disponen elementos de pequeña dimensión en dirección normal a las respectivas superficies para la correcta aplicación del modelo de turbulencia. Por ejemplo, cerca de los álabes del impulsor la malla tiene 15 capas de prismas de $0.1 \mathrm{~mm}$ de espesor cada una, tal como se observa en el detalle de la Fig. 4. Con estos parámetros se alcanzaron resultados con independencia de la malla. 


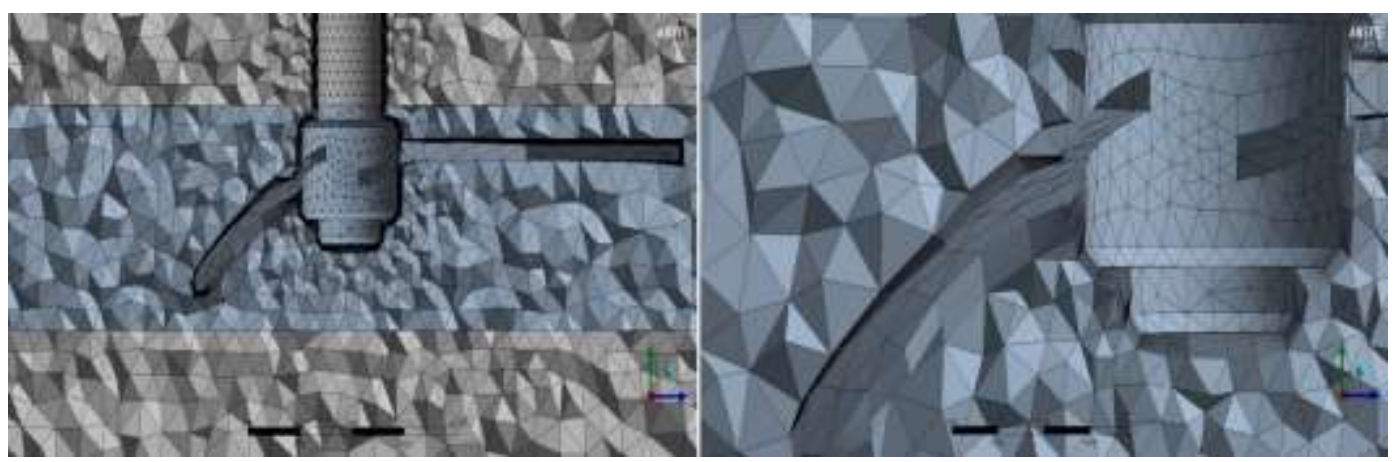

Fig. 4: a) Detalle de la malla cerca del impulsor. b) Detalle de la malla cerca de un álabe.

\section{Condiciones de contorno}

Se fijan once valores de velocidad del impulsor en el rango de 200 a $700 \mathrm{rpm}$ y se realizan simulaciones en estado estacionario para cada uno de estos valores. En el dominio rotativo se establecen las superficies con velocidades prescritas, asociadas a la velocidad angular del impulsor. Además, todas las superficies sólidas del tanque, deflectores y eje se consideran como paredes sin deslizamiento para el fluido. La superficie superior del dominio estacionario está sometida a presión atmosférica. Se establece un volumen ocupado por aire en la parte superior del tanque, prescribiendo un valor inicial de fracción de volumen de líquido de 0; en el resto del volumen, ocupado por la mezcla, se prescribe un valor inicial de fracción de volumen de líquido de 1.

\section{RESULTADOS}

Tal como se ha señalado con respecto al modelo de turbulencia, este ofrece buenos resultados a condición de que en las primeras capas cerca de las superficies sólidas se obtenga altura adimensional $y^{+} \approx 1$. Con la malla descrita, se ha obtenido un valor promedio en las simulaciones de $y^{+} \approx 0.487$. La validación se ha basado en la comparación del torque en el eje del impulsor determinado mediante simulación con el correspondiente valor obtenido experimentalmente. Para generalizar la comparación de resultados, a partir de estos se grafica la curva de Potencia que relaciona los números adimensionales de Potencia y de Reynolds, respectivamente (Kresta et al., 2016):

$$
\begin{aligned}
& N p=\frac{P}{\rho N^{3} D^{5}} \quad \mathrm{y} \\
& R e=\frac{\rho N D^{2}}{\mu}
\end{aligned}
$$

donde: $P$ es la potencia consumida (W), $N$ la velocidad angular (rev/s), $D$ es el diámetro del impulsor (m), $\rho$ es la densidad $\left(\mathrm{kg} / \mathrm{m}^{3}\right)$ y $\mu$ viscosidad aparente que, para el modelo de Bingham utilizado, toma el valor constante del índice de consistencia $K=0.065 \mathrm{~kg} / \mathrm{m} \cdot \mathrm{s}$ (Harnby, 2000; Kresta et al., 2016).

\section{Fracción de volumen y distribución de velocidades}

Las imágenes de la Fig. 5 presentan características del comportamiento a 700 rpm, cuando el flujo está en régimen turbulento plenamente definido. En la Fig. 5a se observa la distribución de la fracción de volumen en un plano de corte longitudinal del tanque. En general, se pueden diferenciar dos grandes zonas ocupadas por el aire en la parte superior y la mezcla en el interior del tanque. Se puede apreciar en detalle, que en el interior del tanque hay zonas de mezcla con presencia de aire; este tiende a ingresar a la mezcla alrededor del eje del impulsor. Esta es un efecto real, asociado a la tendencia circulatoria del fluido a altas velocidades como la mostrada, que es contrarrestada por la presencia de los deflectores. En la Fig. $5 b$ se observan las líneas de corriente en un plano de corte longitudinal. De acuerdo con el patrón característico del flujo originado por impulsores de tipo axial, se aprecia que la mezcla se acelera al pasar por el impulsor desde la parte superior hacia el fondo del tanque, desde el cual rebota subiendo cerca de las paredes hacia la superficie libre, para luego descender nuevamente por la parte central cercana al eje del impulsor. En la Fig. $5 \mathrm{c}$ se muestra la distribución de vectores de velocidad en un plano de corte transversal a una altura de $200 \mathrm{~mm}$ desde el fondo del tanque; esto es, ligeramente por encima del impulsor. Se observa la tendencia radial del flujo en zonas cercanas al impulsor. En zonas próximas a la pared del tanque se aprecia la tendencia tangencial del flujo y el efecto de los deflectores al interrumpir esta tendencia circulatoria y desviar el fluido hacia la zona central donde está el impulsor, favoreciendo con ello que predomine el flujo axial y mejore el proceso de mezclado. 

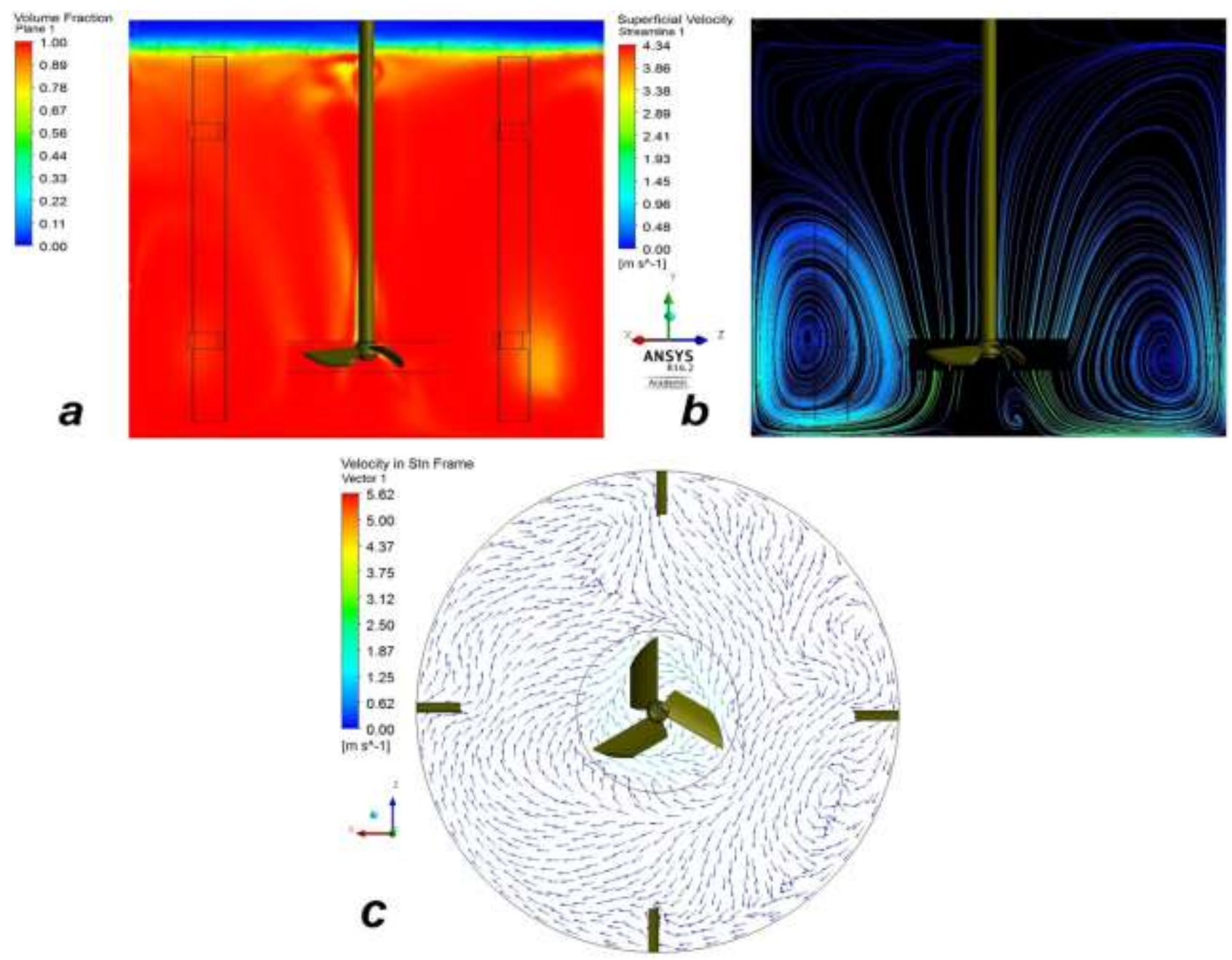

Fig. 5: (a) Fracción volumétrica, (b) líneas de corriente y (c) distribución de vectores de velocidad.

Curva de torque en función de la velocidad angular

En la Figura 6 se grafica la variación del torque en el eje del impulsor obtenido mediante simulación con respecto a la velocidad angular. Como se puede apreciar, el torque tiene un comportamiento creciente con respecto a la velocidad angular. Se observa que cerca de $200 \mathrm{rpm}$ el torque tiene una variación poco significativa, sin embargo a medida que aumenta la velocidad el aumento del torque es mucho más pronunciado.

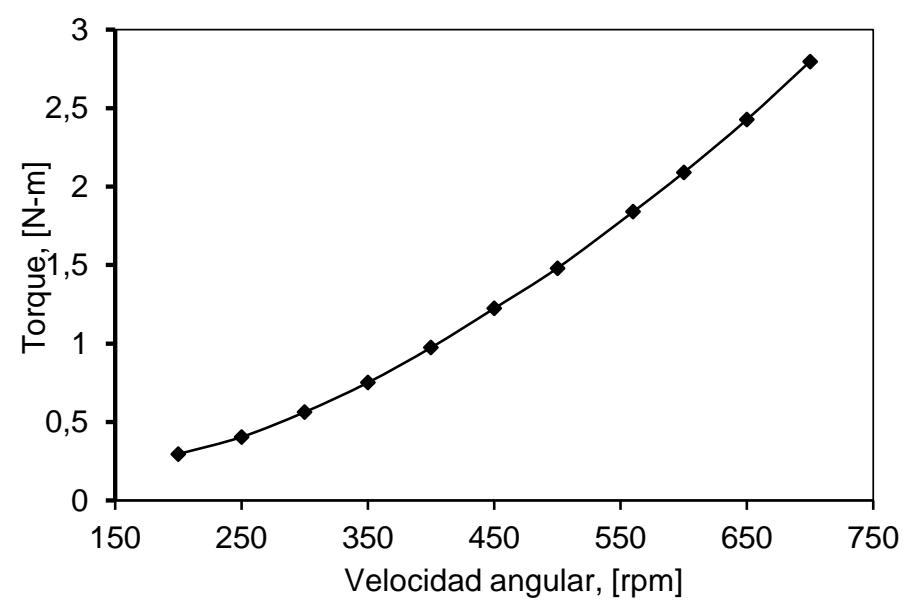

Fig. 6. Torque en función de la velocidad angular del impulsor 
En la Tabla 1 se muestran los valores de torque obtenidos mediante simulación y los determinados experimentalmente, $T_{\text {simulación y }} T_{\text {experimental }}$ respectivamente, la discrepancia entre ellos es del orden del $10 \%$. A partir de los valores de torque y de la velocidad angular se calculan los números de Potencia, presentados en la misma tabla.

Tabla 1: Re y Np para distintas velocidades angulares del impulsor

\begin{tabular}{|c|c|c|c|c|c|}
\hline$N$ & $T_{\text {simulación }}$ & $T_{\text {experimental }}$ & $R e$ & $N p_{\text {simulación }}$ & $N p_{\text {experimental }}$ \\
\hline$[r p m]$ & {$[N \cdot \mathrm{m}]$} & {$[N \cdot \mathrm{m}]$} & {$[-]$} & {$[-]$} & {$[-]$} \\
\hline 200 & 0.29 & 0.27 & 3000 & 0.42 & 0.38 \\
\hline 250 & 0.40 & 0.42 & 3750 & 0.36 & 0.38 \\
\hline 300 & 0.56 & 0.62 & 4500 & 0.35 & 0.38 \\
\hline 350 & 0.75 & 0.81 & 5260 & 0.34 & 0.38 \\
\hline 400 & 0.97 & 1.11 & 6010 & 0.34 & 0.39 \\
\hline 450 & 1.22 & 1.40 & 6760 & 0.34 & 0.39 \\
\hline 500 & 1.48 & 1.70 & 7510 & 0.33 & 0.38 \\
\hline 560 & 1.84 & 2.09 & 8410 & 0.33 & 0.38 \\
\hline 600 & 2.09 & 2.41 & 9020 & 0.32 & 0.38 \\
\hline 650 & 2.43 & 2.80 & 9760 & 0.32 & 0.37 \\
\hline 700 & 2.80 & 3.15 & 10520 & 0.32 & 0.36 \\
\hline
\end{tabular}

\section{Curva de potencia obtenida mediante simulación y comparación con resultados experimentales}

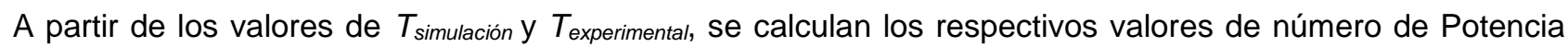

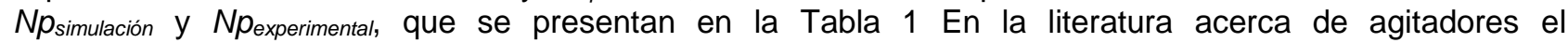
comportamiento característico de los impulsores se representa mediante la denominada curva de Potencia $R e$ vs. Np, en términos de los números adimensionales de Reynolds y de Potencia (Paul et al., 2004). La figura 7 muestra la curva de Potencia del impulsor determinada mediante simulación y los resultados obtenidos mediante experimentación, con la finalidad de poder hacer comparaciones generales. Es así que, como referencia se han digitalizado y graficado también curvas representativas de impulsores hydrofoil disponibles en la literatura (Harnby et al.,2000).

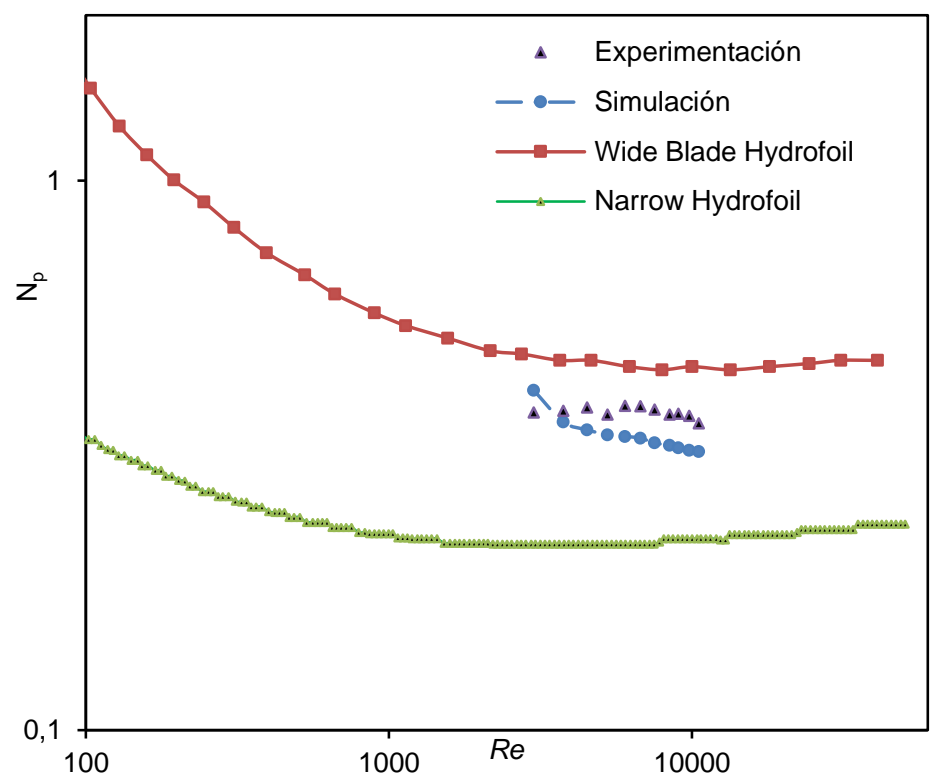

Fig. 7: Comparación de las curvas de potencia obtenidas mediante simulación y resultados experimentales. 
Como se puede observar, hay buena concordancia entre los resultados determinados mediante simulación y los obtenidos experimentalmente. Para altos valores de $R e$, correspondientes a régimen turbulento, la curva obtenida mediante simulación tiende asintóticamente al valor $N p$ de 0.32 ; esta tendencia ocurre de manera clara a partir $R e \approx 5000$, correspondiente a velocidades angulares superiores a $350 \mathrm{rpm}$. El valor de $\mathrm{Np}$ en régimen turbulento es importante para el diseño y operación de agitadores; en la literatura se citan valores referenciales para diferentes tipos de impulsores. El valor obtenido mediante la simulación en el estudio del agitador prototipo, concuerda bastante bien con las referencias incluidas en la figura y con el valor de 0.31, citado para impulsores tipo hydrofoil A310 (Kresta et al., 2016).

De la expresión (5) se desprende que la tendencia asintótica del número de Potencia hacia un valor constante, está asociada a un comportamiento de la potencia consumida que tiende a ser proporcional al cubo de la velocidad angular. Por esta razón, en aplicaciones de mezclado de fluidos es conveniente determinar un valor de velocidad angular de operación que permita una suficiente homogeneidad de la mezcla. Más allá de esta condición de operación, el aumento de la velocidad angular se traduce en un excesivo incremento del consumo de potencia, sin mejora significativa del proceso. Este es un tema de investigación de crucial importancia para aplicaciones industriales.

\section{CONCLUSIONES}

Los resultados del análisis del comportamiento del impulsor tipo hydrofoil de flujo axial realizado mediante simulación numérica presentan muy buena concordancia con los resultados experimentales; la discrepancia obtenida es del orden del 10\%. Se concluye que el valor del Número de Potencia $(N p)$ converge a 0.32 para valores del Número de Reynolds Re en régimen turbulento. El valor obtenido concuerda con el reportado en la literatura para impulsores hydrofoil A310 y la curva de potencia se ajusta a las curvas de referencia para este tipo de impulsores. Por otro lado, las líneas de corriente permiten observar que el flujo generado por el impulsor hydrofoil en el agitador corresponde al patrón típico de impulsores axiales. Asimismo, los vectores de velocidad en planos de corte transversales muestran el efecto de los deflectores, que evitan el flujo circulatorio, favorecen el desarrollo del flujo axial y la mejora del proceso de mezcla.

\section{AGRADECIMIENTOS}

Este artículo reporta resultados parciales de los trabajos de investigación para abordar el diseño de agitadores en colaboración con la empresa local FabTech S.A.C. Los autores agradecen al Fondo para la Innovación, la Ciencia y Tecnología (FINCyT) del Perú, por el financiamiento del proyecto desarrollado por el grupo INACOM de la PUCP, mediante el Convenio № 104-FINCyT-FIDECOM-PIPEA_2011. Asimismo, agradecen muy especialmente al Dr. Elder Mendoza Orbegoso, por sus valiosas discusiones.

\section{REFERENCIAS}

ANSYS Inc, Modeling Guide \& Theory Guide 17.2, USA (2016)

Aubin, J., Fletcher, D.F. y Xuereb, C., Modeling turbulent flow in stirred tanks with CFD: the influence of the modeling approach, turbulence model and numerical scheme, Exp. Th. and Fluid Science 28, 431-445 (2004)

Constantinescu, V. N., Laminar Viscous Flow, Springer-Verlag, New York, USA (1995)

Fernández, J. M., Técnicas Numéricas en Ingeniería de Fluidos, Departamento de Energía, Mecánica de Fluidos, Universidad de Oviedo, Editorial Reverté, España (2012)

Gabriele, A., Nienow, A. y Simmons, M., Use of angle resolved PIV to estimate local specific energy dissipation rates for up - and down-pumping pitched blade agitators in a stirred tank, Chemical engineering Science, 64, 126-143 (2009)

Gidaspow, D., Multiphase Flow and Fluidization, Academic Press, Boston, MA, USA (1994)

Harnby, N., Edwards, M. F. y Nienow, A. W., Mixing in the Process Industries, Second Edition, Oxford: Butterworth Heinemann, England (2000)

Ibrahim, S., Jasnin, S.N., Wong, S.D., y Baker, I.F., Zwietering's equation for the suspension of porous particles and the use of curved blade impellers, International Journal of Chemical Engineering, vol. 2012, 13 pp., doi:10.1155/2012/749760 (2012)

Jaworski, Z. y Zakrzewska, B., Modelling of the turbulent wall jet generated by a pitched blade turbine impeller, The effect of turbulence model, Trans IChemE, 80 Part A, 846-854 (2002) 
Joshi, J.B. y otros 6 autores, CFD simulation of stirred tanks: Comparison of turbulence models. Part I: Radial flow impellers, Canadian J. of Chemical engineering, 89, 23- 82 (2011)

Kehn, R.O., How computational fluid dynamics is applied to mixer design (en línea), Acceso: Mayo 9 de 2015, http://www.processingmagazine.com/articles/print/125490, Processing, USA (2013)

Kim, S. y Choudhury D., A Near Wall Treatment using Wall Functions Sensitized to Pressure Gradient, ASME FED, vol. 217, Separated and Complex Flow, ASME, USA (1995)

Kresta, S., Etchells III, A., Dickey, D. y Atiemo-Obeng, V., Advances in Industrial Mixing, John Wiley \& sons, Inc. publication, NJ, USA (2016)

Luo, J. Y., Issa R. I. y Gosman A. D., Prediction of Impeller-Induced Flows in Mixing Vessels using Multiple Frames of Reference, IChemE Symposium Series, 136, 549-556 (1994)

Montante, G., Micale G., Magelli F., Brucato A., Experiments and CFD predictions of solid particle distribution in a vessel agitated with four pitched blade turbines, Trans IChemE, 79 Part A, 1005-1010 (2001)

Paul, E., Atiemo-Obeng, V., Kresta, S., Handbook of Ind. Mixing, John Wiley \& sons, Inc. publication, NJ, USA (2004)

Patankar, S.V., Computational modelling of flow and heat transfer in industrial applications, Int. J. of Heat and Fluid Flow, 23, 222-231 (2002)

Qingbai, C. y Gance, D., Flooding characteristics of hydrofoil impeller in a two- and three-phase stierred tank, Chinese J. of Chemical engineering, 18 (3), 355-361 (2010)

Shah, S.R., Jain, S.V., Patel R.N., Lakhera V.J., CFD for centrifugal pumps: a review of the state of the art, Procedia Engineering, 51, 715-720 (2013)

Spogys, N. y Nunhez, J., Optimizing a hydrofoil for industrial use, Procedia Eng., 138, 403-412 (2016)

Versteeg, H. K. y Malalasekera, W., An Introduction to Computational Fluid Dynamics: The Finite Volume Method, Pearson Education, England (2007)

Wu, J., Graham, L., Nguyen, B. y Nabil, M., Energy efficiency study on axial flow impellers, Chemical Engineering and Processing, 45, 625-632 (2006)

Wu, J., Graham L., Wang S. y Parthasarathy R., Energy efficiency slurry holding and transport, Minerals Engineering, 23, 705-712 (2010a)

Wu, J., B. Nguyen y L. Graham, Energy efficiency high solids loading agitation for the mineral industry, Canadian Journal of Chemical Engineering, 88, 287-294 (2010b)

Yang F. y Zhou S., Free surface turbulent flow in an unbaffled stirred tank: Detached Eddy simulation and VOF study, Chem. Biochem. Eng. Q., 29 (3), 395-403 (2015)

Yapici, K., Numerical Investigation of the effect of the Rushton type turbine design factors on agitated tank flow characteristics, Chemical Engineering and Processing: Process Intensification, 47 (8) 1340-1349 (2008)

Zwietering, T.N, Suspension of solids in liquid by agitators, Chemical Engineering Science, 8, 244-253 (1958) 